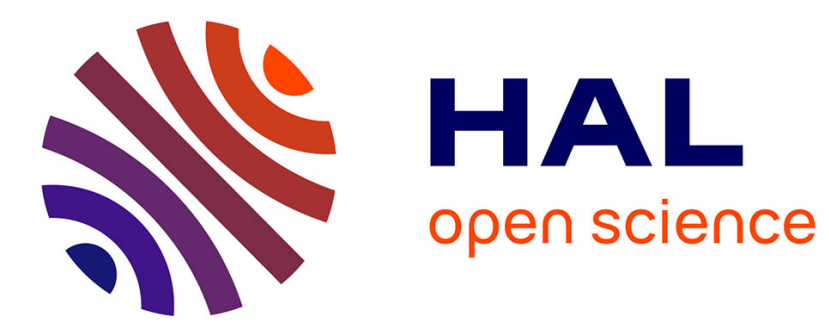

\title{
Preasymptotic charge oscillations around 3d impurities in aluminium
}

V. Zlatić, G. Grüner

\section{To cite this version:}

V. Zlatić, G. Grüner. Preasymptotic charge oscillations around 3d impurities in aluminium. Journal de Physique Lettres, 1977, 38 (3), pp.87-89. 10.1051/jphyslet:0197700380308700 • jpa-00231333

\section{HAL Id: jpa-00231333 https://hal.science/jpa-00231333}

Submitted on 1 Jan 1977

HAL is a multi-disciplinary open access archive for the deposit and dissemination of scientific research documents, whether they are published or not. The documents may come from teaching and research institutions in France or abroad, or from public or private research centers.
L'archive ouverte pluridisciplinaire HAL, est destinée au dépôt et à la diffusion de documents scientifiques de niveau recherche, publiés ou non, émanant des établissements d'enseignement et de recherche français ou étrangers, des laboratoires publics ou privés. 


\title{
PREASYMPTOTIC CHARGE OSCILLATIONS AROUND 3d IMPURITIES IN ALUMINIUM
}

\author{
V. ZLATIĆ and G. GRU̇NER $\left(^{*}\right)$ \\ Institute of Physics, University of Zagreb, Zagreb, Yugoslavia \\ (Reçu le 4 octobre 1976, révisé le 21 décembre 1976, accepté le 3 janvier 1977)
}

\begin{abstract}
Résumé. - L'objet de cet article est de calculer dans le modèle d'Anderson la perturbation de densité électronique autour de certaines impuretés $3 \mathrm{~d}$ dans l'aluminium. L'atténuation de l'amplitude des oscillations de Friedel au voisinage de l'impureté est discutée dans le modèle des fluctuations de spins localisés. La dépendance en température de $\Delta \rho(r)$ est aussi considérée.
\end{abstract}

\begin{abstract}
The charge density oscillations in Al 3d alloys are discussed and the observed values, which are strongly reduced with respect to the values predicted by Friedel's expression, are explained using the localised spin fluctuation approximation of the Anderson model. Temperature dependence of $\Delta \rho$ is discussed as well.
\end{abstract}

Formation of the long range spin polarization around the Kondo impurities has been extensively studied an is well understood [1-3] but relatively little is known about the effect of the many-body Kondo state on the charge polarization of the host. In this letter we discuss some new aspects of the charge polarization in Kondo alloys and show that recent experimental data on aluminium based transition metal alloys can be explained in the LSF approximation of the Anderson model.

A number of recent nuclear magnetic resonance and nuclear quadrupole resonance studies on Al-3d alloys revealed that close to the impurity the amplitude of the conduction electron charge density is strongly reduced with respect to the values predicted by Friedel's asymptotic expression [3-7]. This preasymptotic reduction of the charge density, $\Delta \rho(r)$, reflects the energy dependence of the resonant phase shift, neglected in the original derivation of the asymptotic formula. If $\Delta \rho_{\mathrm{F}}(r)$ denotes the Friedel values, measurements on the first few neighbouring shells of the impurity show that the order of magnitude of $\Delta \rho(r) / \Delta \rho_{\mathrm{F}}(r)$ is given by $\Delta / \varepsilon_{\mathrm{F}}$, where $\Delta$ is the width of the virtual bound state and $\varepsilon_{\mathrm{F}}$ is the Fermi energy. One can argue that the relevant energy scale over which the resonant phase shift varies from 0 to $\pi$ is proportional to $\Delta$. With this assumption, the one-electron approximation explains most of the experimental results on $\mathrm{Al} 3 \mathrm{~d}$ alloys [5]. The only exceptions are AlMn and $\underline{\mathrm{A}} \mathrm{l} \mathrm{Cr}$

(*) Permanent address : Central Research Institute for Physics, Budapest, Hungary. where the experimental data remain anomalously low.

Measurements of the temperature dependence of the charge oscillations in $\mathrm{AlMn}$ and $\mathrm{AlCr}$ have shown [7] that in the asymptotic region $\Delta \rho(T)$ is strongly temperature dependent and that it exhibits a universal behaviour as a function of $T / T_{\mathrm{K}}$ where $T_{\mathrm{K}}$ is the LSF (or Kondo) temperature determined from thermal or transport properties. If follows [8] that the relevant energy scale in the asymptotic region is proportional to $T_{\mathrm{K}}$. However, if $\Delta \rho(r)$ in the preasymptotic region is calculated in the one-electron approximation with $\Delta \simeq T_{\mathrm{K}}$, the values obtained are much below the experimental data [3,5]. Such a difference in the asymptotic and preasymptotic behaviour indicates that the complete description of the radial and temperature dependence of the charge density oscillations in dilute alloys requires a detailed knowledge of both the low and high energy sections of the electronic spectrum $\left(\varepsilon \sim T_{\mathrm{K}}\right.$ and $\left.\varepsilon \sim \Delta\right)$. Any single parameter theory should not be expected to give the complete answer to questions related to the charge and spin density polarization.

In this note we show that the localized spin fluctuation (LSF) approximation of the Anderson hamiltonian, used mostly in connection with the low frequency properties of nearly magnetic alloys [9], explains also the nuclear quadrupole resonance data on AlMn and $\mathrm{AlCr}$. The approximation is based on the phenomenological description of the local elementary excitations (LSF) and on the assumption that the dominant contribution to the d-electrons self energy 
comes from its scattering on the LSF. It has been shown [10] that in the LSF approximation the virtual bound state associated with the nearly magnetic impurity has a triply peaked structure : a narrow, temperature dependent peak (width of the order of $T_{\mathrm{K}}$ ) around the Fermi level and two broad wings $(\sim \Delta)$ which depend only weakly on temperature. Temperature variation of the charge density at large distances from the impurity follows the behaviour of the central peak, while the preasymptotic damping is influenced by the total structure of the vbs.

Consistency of a triply peaked structure of the vbs with both the local and transport properties was emphasised first by Grüner and Zawadowski [8]. The perturbation-theoretical results for the Anderson hamiltonian [11] have also shown that the local density of states is triply peaked. (Actually, Yamada's results [11] are strikingly similar to the phenomenological LSF results.) Thus, one can argue that the charge density oscillations obtained in the LSF approximation are not different from what one would obtain using the rigorous perturbation theory of Yosida and Yamada [11].

In order to calculate $\Delta \rho(r)$ of an LSF alloy, at point $r$ and temperature $T$, we start from the Anderson model in which $\Delta \rho(r, T)$ is given by (we follow closely Anderson [12]) :

$$
\begin{aligned}
\Delta \rho=\frac{k_{\mathrm{B}} T}{(2 \pi)^{6}} \lim _{\tau \rightarrow 0} \sum_{\varepsilon_{n}} & \times \int \mathrm{d}^{3} \mathbf{k}_{1} \mathrm{~d}^{3} \mathbf{k}_{2} G^{0} \mathbf{k}_{1} G_{\mathbf{k}_{2}}^{0} T_{\mathbf{k}_{1} \mathbf{k}_{2}} \\
& \times \exp \left[i\left(\mathbf{k}_{1}-\mathbf{k}_{2}\right) \mathbf{r}-i \varepsilon_{n} \tau\right]
\end{aligned}
$$

$G_{\mathbf{k}}^{0}\left(i \varepsilon_{n}\right)$ is the unperturbed Green function of conduction electrons, $T_{\mathbf{k}_{1} \mathbf{k}_{2}}$ is the scattering matrix and $\varepsilon_{n}$ is the Fermion frequency. In the Anderson model one has

$$
T_{\mathbf{k}_{1} \mathbf{k}_{2}}=V_{k_{1} \mathrm{~d}} G_{\mathrm{d}}\left(i \varepsilon_{n}\right) V_{\mathrm{d} k_{2}}
$$

where $G_{\mathrm{d}}\left(i \varepsilon_{n}\right)$ is the d-electrons Green function, $G_{\mathrm{d}}=\left(i \varepsilon_{n}-E_{\mathrm{d}}+i \Delta-\Sigma_{\mathrm{d}}\right)^{-1}$ and $E_{\mathrm{d}}$ and $\Delta$ are the position and the width of the unperturbed vbs. The self energy correction to the d-propagator due to the LSF, is

$$
\Sigma_{\mathrm{d}}\left(i \varepsilon_{n}\right)=T \sum_{m} G_{\mathrm{d}}\left(i \varepsilon_{n}+i \varepsilon_{m}\right) \chi_{\mathrm{d}}\left(i \varepsilon_{m}\right)
$$

where

$$
\chi_{\mathrm{d}}=\pi \Delta^{2} /\left(\left|\varepsilon_{m}\right|+T_{\mathrm{K}}\right)
$$

is the LSF propagator and $T_{\mathrm{K}}$ the phenomenological lifetime. If we assume (following Anderson [12]) that $V_{k \mathrm{~d}}$ couples the d-state to the $l=2$ spherical component of the conduction states only, the $k$ integrations in (1) can be performed analytically. We mention here that $\Sigma_{\mathrm{d}}$ calculated in the LSF model agrees well with the results of ref. [11].
For a level of degeneracy $(2 l+1)$, we obtain

$$
\begin{aligned}
& \Delta \rho(r, T)=-\frac{2 l+1}{4 \pi^{2}} \frac{\Delta}{\varepsilon_{\mathrm{F}}} \frac{1}{\left(k_{\mathrm{F}} r\right)^{2}} \times \\
& \times \operatorname{Im} \int \mathrm{d} \varepsilon f(\varepsilon) g_{l}^{2}(\varepsilon) \frac{1}{\varepsilon-E_{\mathrm{d}}+i \Delta-\Sigma_{\mathrm{d}}}
\end{aligned}
$$

Here, $g_{l}(\varepsilon)$ is a simple analytical expression which is for our purposes sufficiently well approximated by (see also Alloul [3])

$$
g_{l}(\varepsilon)=-\exp i(k r-l \pi / 2),
$$

where

$$
k=\left\{2 m^{*}\left(\varepsilon+\varepsilon_{\mathrm{F}}\right)\right\}^{1 / 2} .
$$

All the symbols have their usual meaning and sum over $\varepsilon_{n}$ has been evaluated by standard methods. When $\Sigma_{\mathrm{d}}$ is negligible (the case of all the Al $3 \mathrm{~d}$ alloys except AlMn and $\mathrm{AlCr}$ ) charge density given by equation (3) can be evaluated exactly [13]. In the case of nearly magnetic impurities, we calculate the selfenergy $\Sigma_{\mathrm{d}}$ in the LSF approximation [10] and evaluate the integral for $\Delta \rho$ (in the preasymptotic region) numerically. Since the virtual bound state associated with $\mathrm{AlMn}$ and $\mathrm{AlCr}$ is half-full, we put $E_{\mathrm{d}}=0$ and calculate $\Delta \rho$ with $\Delta=1 . \mathrm{eV}$ and $T_{\mathrm{K}}=0.131 \mathrm{eV}$ $\left(T_{\mathrm{K}}=0.393 \mathrm{eV}\right)$. These values of $T_{\mathrm{K}}$ are obtained by analyzing the resistivity data on AlMn and $\mathrm{AlCr}$ alloys [14].

At very large distances function $g_{l}(\varepsilon)$ oscillates rapidly and the integral for $\Delta \rho$ differs from zero only in a very small energy interval close to $\varepsilon_{\mathrm{F}}$. Replacing $G_{\mathrm{d}}(\varepsilon)$ by its value at the Fermi level we see that $\Delta \rho(r, T=0)$ reduces to Friedel's asymptotic expression.

In the preasymptotic region the radial dependence of $\Delta \rho(r, T=0)$ is obtained by numerical computation and is shown in figure 1. For the first few neighbouring

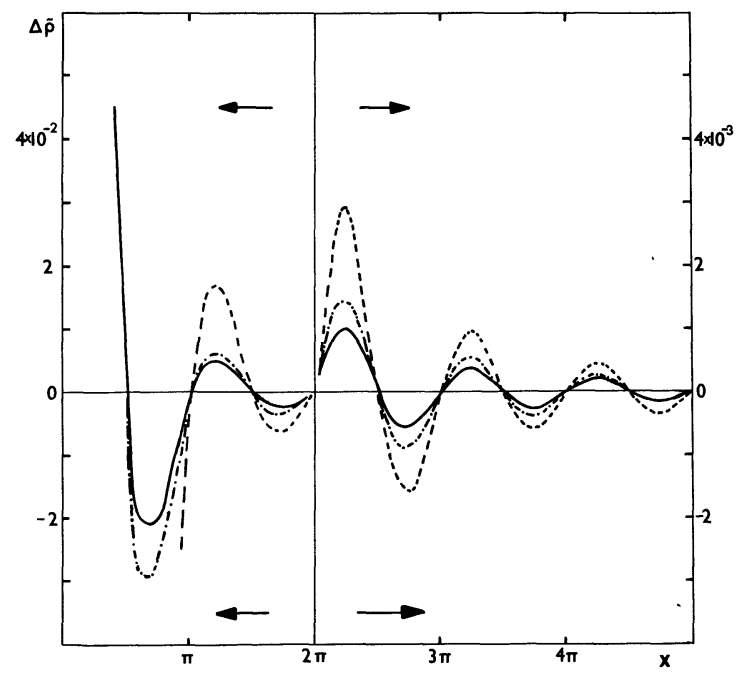

Fig. 1. $-\Delta \widetilde{\rho}=\Delta \rho(r) / 4 \pi^{2}(2 l+1)$ plotted as a function of distance from the impurity in units $x=k_{\mathrm{F}} r$ (full line). Broken line is the corresponding Friedel's asymptotic expression and dotted line is the H-F result obtained with $\Delta=1.0 \mathrm{eV}$ and $E_{\mathrm{d}}=0$. 
impurity shells in aluminium $\left(k_{\mathrm{F}} r=5,7,9\right.$, etc. $)$ the average value of $\Delta \rho / \Delta \rho_{F}$, is about $\Delta \rho / \Delta \rho_{F}=0.3$ for AlMn. The LSF results for the preasymptotic damping of $\Delta \rho$ are similar to the Hartree-Fock results of Mezei and Grüner [13] but for a given pair of $E_{\mathrm{d}}$ and $\Delta$, the amplitude of $\Delta \rho(r)$ obtained in the H-F . theory is substantially larger than the corresponding LSF amplitude. In particular, $\Delta \rho_{\mathrm{LSF}}$ calculated with $\Delta=1 \mathrm{eV}, \quad E_{\mathrm{d}}=0$ and $T_{\mathrm{K}}=0.131 \mathrm{eV}$ $\left(T_{\mathrm{K}}=0.393 \mathrm{eV}\right)$ is approximatively equal to $\Delta \rho_{\mathrm{HF}}$ calculated with $\Delta=0.4 \mathrm{eV}$ and $E_{\mathrm{d}}=0(\Delta=0.6 \mathrm{eV})$.

In figure 2 the temperature dependence of the charge density oscillations is plotted for various values of $r$, normalized to unity at $T=0 \mathrm{~K}$. When $r$ tends to infinity $\Delta \rho(T)$ becomes a universal function [14] of $T / T_{\mathrm{K}}$. For arbitrary distance, $\Delta \rho(r, T)$ decreases parabolically from the $T=0$ value,

$$
\Delta \rho(r, T)=\Delta \rho(r, 0)\left[1-\left(\frac{T}{\theta_{r}}\right)^{2}\right]
$$

where $\theta_{r}$ is the characteristic temperature which is decreasing with increasing $r$. At infinity one has $\theta_{r}=\left(2 / \pi^{2}\right)^{1 / 2} T_{\mathrm{K}}$. In the case of AlMn, $\theta_{r}=1500 \mathrm{~K}$ at the nearest neighbours distance, while $\theta_{\infty}=684 \mathrm{~K}$. Above temperatures of the order of $T_{\mathrm{K}} / 2 \pi, \Delta \rho(T)$

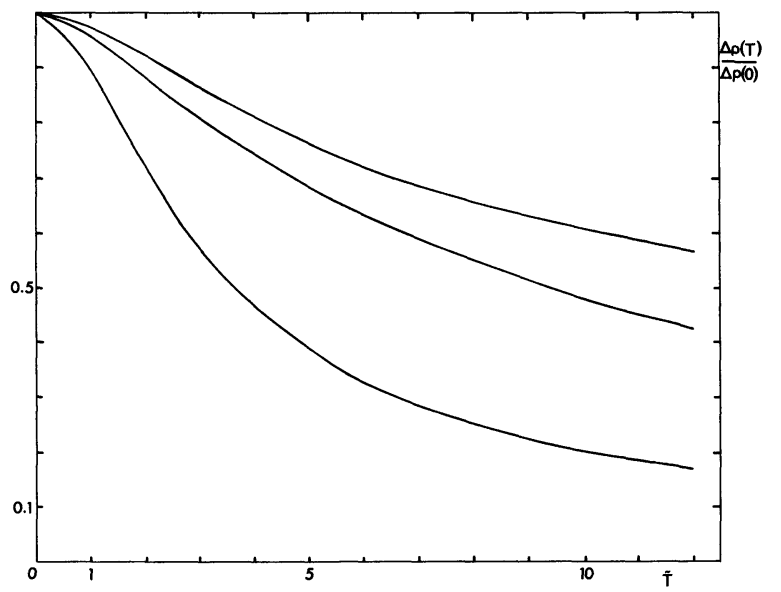

FIG. 2. $-\Delta \rho$ as a function of reduced temperature $T=2 T / T_{\mathrm{K}}$, normalized to unity at $T=0.0 \mathrm{~K}\left(\Delta=1.0 \mathrm{eV}, E_{\mathrm{d}}=0.0\right.$ and $\left.T_{\mathrm{K}}=0.131 \mathrm{eV}\right)$. Top curve corresponds to nearest neighbours of $\mathrm{Mn}$ in Al. Middle and bottom curve correspond to $k_{\mathrm{F}} r=9$, and $k_{\mathrm{F}} r=\infty$, respectively. becomes a linear function of $T$ and finally, for large enough temperatures, $\Delta \rho(T)$ goes to zero. The smaller the distance from the impurity the weaker the temperature variation of $\Delta \rho$; such a behaviour reflects the change in the energy interval over which the integral for $\Delta \rho$ differs from zero.

Finally, we comment on the experimental situation relevant to our results. From the high precision nuclear quadrupole resonance experiments on $\mathrm{Al} 3 \mathrm{~d}$ alloys [5, 6], the detailed values of the conduction electrons charge density around $3 \mathrm{~d}$ impurity are now available. Berthier and Minier [5] were able to interpret the experimental data in terms of the Mezei and Grüner theory [13], but the width of the virtual bound state they needed to fit the AlMn and $\mathrm{AlCr}$ data $(\Delta=0.5 \mathrm{eV})$ was only about one half of the width which fitted all the other $\mathrm{Al} 3 \mathrm{~d}$ alloys $(\Delta=1.0 \mathrm{eV})$ and such a drastic change of the vbs was difficult to understand in the Friedel-Anderson model. From our results it is clear that the preasymptotic damping of $\Delta \rho(r)$ in $\mathrm{AlMn}$ and $\mathrm{AlCr}$ is a consequence of the fluctuations of the local magnetization at the impurity d-level. The values of $\Delta \rho_{\mathrm{LSF}}$ corresponding to the first few neighbouring shells agree, in these alloys, surprisingly well with the experimental data (see discussion following figure 1). If we vary the position of the vbs, $E_{\mathrm{d}}$, keeping $\Delta=1.0 \mathrm{eV}$ throughout the $3 \mathrm{~d}$ series, an excellent agreement between the measured and calculated charge density is obtained for all the Al 3d alloys.

As regards the temperature dependence of $\Delta \rho$ the observed behaviour in the asymptotic region agrees with the LSF results [7] but further experiments are needed to obtain the informations on $\Delta \rho(T)$ in the preasymptotic region. The fact that in AlMn the quantity $\Delta \rho(r)$ is predicted to be temperature dependent both for small and large distances is associated to the fact that $\Delta / T_{\mathrm{K}}$ is not too small a number. (See also discussion in ref. [2].)

In conclusion, we have shown that the screening of a transition metal impurity in a normal metal host can be simply explained by the Friedel-Anderson model. In order to obtain the quantitative agreement with the experimental data on nearly magnetic alloys, dynamical properties of d-electrons have to be taken into account.

\section{References}

[1] Boyce, J. B. and Slichter, C. P., Phys. Rev. Lett. 32 (1974) 61.

[2] Alloul, H. J., AIP Conference Proceedings No 29, 300 (1975) (Eds. J. J. Becker, G. H. Lander and J. J. Rhyne) IsHII, H., Prog. Theor. Phys. 55 (1976) 1373.

[3] Alloul, H. J., J. Phys. F 4 (1974) 1501.

[4] Grüner, G., Solid State Commun. 10 (1972) 1033.

[5] Berthier, C. and Minier, M., J. Phys. F 3 (1973) 1169.

[6] Stiles, J. A., Kaplan, N. and Williams, D. Ll., J. Phys. F 5 (1975).

[7] Grüner, G. and Hargitai, C., Phys. Rev. Lett. 26 (1971) 772.

[8] Grüner, G. and Zawadowski, R., Solid State Commun. 11 (1972) 663.
[9] Rivier, N. and Zuckermann, M. J., Phys. Rev. Lett. 21 (1968) 904.

[10] Zlatić, V., Grüner, G. and Rivier, N., Solid State Commun. 14 (1974) 639.

[11] Yamada, K., Prog. Theor. Phys. 53 (1975) 970.

[12] ANDERson, P. W., Theory of Magnetism in Transition Metals, Proc. 1966 Varenna Summer School, ed. W. Marshal (Academic P., N.Y.) 1967.

[13] Mezei, F. and Grüner, G., Phys. Rev. Lett. 29 (1972) 1465.

[14] Rivier, N. and Zlatić, V., J. Phys. F 2 (1972) L 87. 